\section{Increasing Rates of Methicillin-Resistant Staphylococcus aureus in Academic Hospitals: A Result of Active Surveillance?}

To the Editor-David et al ${ }^{1}$ recently reported that the prevalence of methicillin-resistant Staphylococcus aureus (MRSA) hospitalizations has significantly increased in US academic medical centers since 2003. They based this conclusion on analysis of billing data from the University Health Consortium. Their findings seemingly disagree with those of other investigators. Recently, Landrum et $\mathrm{al}^{2}$ reported a significant decline in the incidence of invasive MRSA-related bloodstream and community-acquired skin and soft-tissue infections in a large and geographically diverse population of military personnel and their families. Microbiologic surveillance data from the Centers for Disease Control and Prevention's (CDC's) National Healthcare Safety Network, ${ }^{3}$ the CDC's Active Bacterial Surveillance Core, ${ }^{4}$ and the Veterans Affairs health system ${ }^{5}$ have also indicated similar decreasing incidence of healthcare-associated MRSA infections.

The approach of David and colleagues was unique; they sought to quantify the total burden of all MRSA-related hospitalizations, whereas other studies have evaluated the incidence of MRSA only in subsets of patients either with specific infection types or with specific locations of infection onset (eg, the intensive care unit). The authors acknowledged the insensitivity of administrative data in identifying MRSA infections, and they attempted to correct for this by performing a sensitivity analysis using surveillance data from one medical center. However, no attempts were made to correct for the low specificity of administrative data for detecting MRSA infections, which occurs because administrative billing codes frequently fail to accurately differentiate active MRSA infection from either colonization with MRSA or history of prior MRSA infection. ${ }^{6}$

We examined data from the Duke Infection Control Outreach Network (DICON) to evaluate trends in MRSA infection rates in community hospitals in 5 states in the southeastern United States. Infection preventionists within DICON hospitals prospectively collected and categorized isolates of MRSA as colonization, hospital acquired, healthcare associated, or community acquired according to standard surveillance definitions. ${ }^{4}$ Complete surveillance data were available from 18 hospitals from 2005 to 2011. Active surveillance practices for MRSA varied by institution, but they generally increased in our network over this period.

The incidence of hospital-acquired MRSA infections in these 18 community hospitals decreased from 0.56 infections per 1,000 patient-days in 2005 to 0.13 infections per 1,000 patient-days in 2011. This downward trend is similar to the observations of others..$^{2-5}$ We then examined the number of nonduplicate MRSA events per unique patient in each calendar year in DICON. We found that the total burden of MRSA, including colonization and invasive infections, increased more than $60 \%$ (2,970 events per year in 2005 to 4,850 events per year in 2011). The annual incidence of invasive infections due to MRSA, including hospital-acquired, healthcare-associated, and community-acquired infections, decreased modestly over this period (Figure 1).

Our surveillance data demonstrate a decline in the incidence of hospital-acquired MRSA infections, similar to trends previously described by others in different surveillance networks who have utilized similar microbiologic surveillance techniques. At the same time, our data demonstrate an increase in the annual burden of MRSA in our network over this period, and we interpret these data with caution. We believe that the apparent increase in the burden of MRSA in DICON was largely driven by increased detection of MRSA colonization; the number of study hospitals performing active surveillance for MRSA increased over this period, and so the potential for detection bias was high. Thus, we cannot with any accuracy distinguish a true increase in MRSA burden from an increase in recognition of MRSA colonization due to changes in surveillance practices. It is very possible that the data from David and colleagues showed increasing total burden of MRSA hospitalizations that was partly attributable to increased detection of colonization due to increased use of active surveillance for MRSA during the study period.

Thus, we have demonstrated that different methodologies

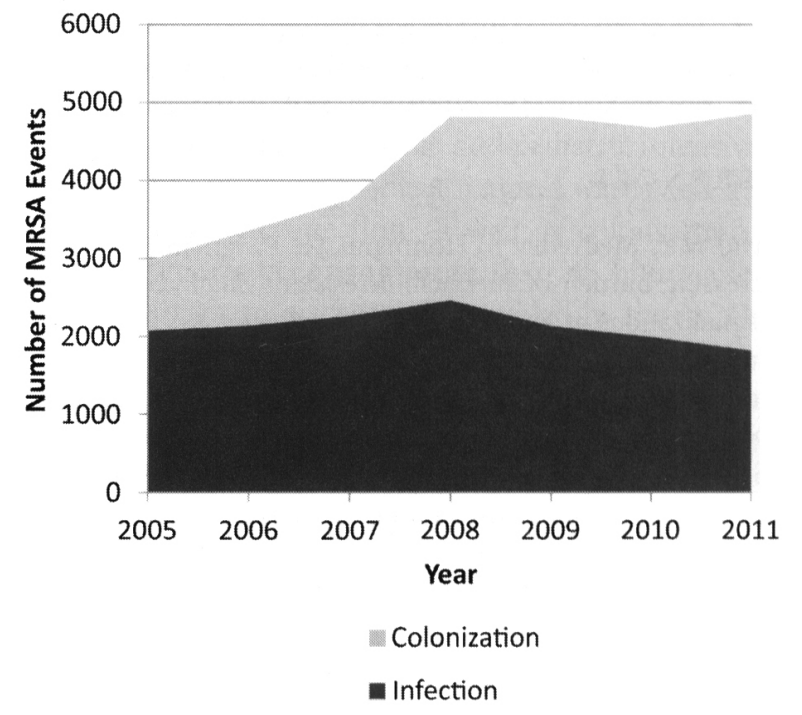

FIGURE 1. Number of methicillin-resistant Staphylococcus aureus (MRSA) events per unique patient per year in 18 Duke Infection Control Outreach Network hospitals from 2005 to 2011. 
may lead to different or even conflicting conclusions about the rates of MRSA infections. We appreciate the efforts of David and colleagues to quantify the total burden of MRSA in a large consortium of hospitals; however, we believe that their use of administrative data lacks the specificity needed to discern true infection from colonization. Thus, their conclusions cannot be directly compared to those of other investigators who have employed microbiologic or surveillance methods to quantify the specific incidence of MRSA infections.

\section{ACKNOWLEDGMENTS}

Financial support. D.J.A. was supported by grant funding from the National Institute of Allergy and Infectious Diseases, National Institutes of Health (K23AI095457).

Potential conflicts of interest. All authors report no conflicts of interest relevant to this article. All authors submitted the ICMJE Form for Disclosure of Potential Conflicts of Interest, and the conflicts that the editors consider relevant to this article are disclosed here.

\section{Sarah S. Lewis, MD; ${ }^{1}$ Rebekah W. Moehring, MD, MPH; ${ }^{1}$ Deverick J. Anderson, MD, MPH; ${ }^{1}$ Daniel J. Sexton, MD, FIDSA; ${ }^{1}$ Luke F. Chen, MBBS, MPH, CIC, FRACP}

Affiliation: 1. Department of Medicine, Division of Infectious Diseases, Duke University Medical Center, Durham, North Carolina; Duke-University of North Carolina Centers for Disease Control and Prevention Epicenter Program, Durham, North Carolina; Duke Program for Infection Prevention and Healthcare Epidemiology, Durham, North Carolina; and Duke Infection Control Outreach Network, Durham, North Carolina.

Address correspondence to Sarah S. Lewis, MD, PO Box 102359, Department of Medicine, Division of Infectious Diseases, Duke University Medical Center, Durham, NC 27710 (sarah.stamps@duke.edu).

Infect Control Hosp Epidemiol 2013;34(1):105-106

(C) 2013 by The Society for Healthcare Epidemiology of America. All rights reserved. 0899-823X/2013/3401-0019\$15.00. DOI: $10.1086 / 668797$

\section{REFERENCES}

1. David MZ, Medvedev S, Hohmann SF, Ewigman B, Daum RS. Increasing burden of methicillin-resistant Staphylococcus aureus hospitalizations at US academic medical centers, 2003-2008. Infect Control Hosp Epidemiol 2012;33(8):782-789.

2. Landrum ML, Neumann C, Cook C, et al. Epidemiology of Staphylococcus aureus blood and skin and soft tissue infections in the US military health system, 2005-2010. JAMA 2012;308(1):50-59.

3. Burton DC, Edwards JR, Horan TC, Jernigan JA, Fridkin SK. Methicillin-resistant Staphylococcus aureus central line-associated bloodstream infections in US intensive care units, 1997-2007. JAMA 2009;301(7):727-736.

4. Kallen $\mathrm{AJ}, \mathrm{Mu} \mathrm{Y}$, Bulens $\mathrm{S}$, et al. Health care-associated invasive MRSA infections, 2005-2008. JAMA 2010;304(6):641-648.

5. Jain $R$, Kralovic SM, Evans ME, et al. Veterans Affairs initiative to prevent methicillin-resistant Staphylococcus aureus infections. $N$ Engl J Med 2011;364(15):1419-1430.

6. Schweizer ML, Eber MR, Laxminarayan R, et al. Validity of $I C D$ -
9-CM coding for identifying incident methicillin-resistant Staphylococcus aureus (MRSA) infections: is MRSA infection coded as a chronic disease? Infect Control Hosp Epidemiol 2011;32(2): $148-154$.

\section{Reply to Lewis et al}

To the Editor-We appreciate the response by Lewis et al $^{1}$ to our recently published report. ${ }^{2}$ We agree that unadjusted administrative and billing data have limitations as a surveillance tool, as we indicated in our report. ${ }^{3}$ However, we must disagree with several points these authors raised.

First, our data spanned the years 2003-2008 from a group of academic medical centers and their hospital affiliates throughout the country. Our data included all inpatient methicillin-resistant Staphylococcus aureus (MRSA) infections. We did not report on trends after 2008. Lewis and colleagues, in contrast, analyzed data on only invasive MRSA infections at just 18 community hospitals in a region of a single state from 2005 to 2011 .

Second, Lewis and colleagues examined only "colonization and invasive infections" in their analyses. As others have shown convincingly, ${ }^{4}$ invasive MRSA infections in the United States declined during the period 2005-2010. We do not dispute this trend in our article. Unlike Lewis and colleagues, in our report we included all MRSA infections, both invasive and noninvasive. It is not clear why Lewis and colleagues would propose any direct comparison or anticipate that the 2 data sets would show the same trends.

Third, Lewis and colleagues stated that we did not attempt to account for the "low specificity of administrative data for detecting MRSA infections." It is true that in deriving estimates of adjusted incidence rates of MRSA infections in University Health Systems Consortium (UHC) centers, we accounted only for the decreased sensitivity of the UHC data. We did not include in these estimates the impact of limitations in the specificity of UHC data. However, we did assess the number of "false positives" captured by UHC data in our validation algorithm. Our method was as follows: counting only a single hospital discharge per person per year, we tabulated every MRSA infection from the University of Chicago Medical Center (UCMC) that was reported to the UHC database from July 1,2004 , through June 30,2005 , and for the years 2006 and 2007. For each UHC-coded MRSA-associated hospital discharge, it was determined whether a MRSA infection was also recorded in the UCMC MRSA Surveillance Project. If no MRSA infection was reported for a UHC-coded MRSA-associated hospital discharge in the UCMC MRSA Surveillance Project data, an infectious diseases physician conducted a medical chart review. If the chart review revealed that there was no MRSA infection, the relevant hospital discharge was categorized being "not a MRSA infection." The 